\title{
Les territoires du secret : confidentialité et enquête dans les mondes pluriels de la sécurité
}

Introduction

Grégory Daho, Emmanuel-Pierre Guittet et Julien Pomarède

\section{(2) OpenEdition \\ Journals}

\section{Édition électronique}

URL : http://journals.openedition.org/conflits/21827

DOI : $10.4000 /$ conflits. 21827

ISSN : $1777-5345$

Éditeur :

CECLS - Centre d'études sur les conflits - Liberté et sécurité, L'Harmattan

\section{Édition imprimée}

Date de publication : 1 décembre 2020

Pagination : $7-17$

ISBN : 978-2-343-21960-8

ISSN : $1157-996 \mathrm{X}$

\section{Référence électronique}

Grégory Daho, Emmanuel-Pierre Guittet et Julien Pomarède, « Les territoires du secret : confidentialité et enquête dans les mondes pluriels de la sécurité », Cultures \& Conflits [En ligne], 118 | été 2020, mis en ligne le 01 décembre 2020, consulté le 31 mars 2021. URL : http://journals.openedition.org/ conflits/21827 ; DOI : https://doi.org/10.4000/conflits.21827 


\section{Les territoires du secret : confidentialité et enquête dans les mondes pluriels de la sécurité}

Introduction

\section{Grégory DAHO, Emmanuel-Pierre GUITTET et Julien POMARÈDE}

Grégory Dabo est maître de conférences en science politique à l'Université Paris 1 Panthéon-Sorbonne, responsable du Master 1 de Relations Internationales et de l'axe de recherche sur les processus d'internationalisation du Centre Européen de Sociologie et de Science Politique (CESSP).

Emmanuel-Pierre Guittet est chercheur associé au Centre d'études sur les conflits, liberté et sécurité (CECLS, France), directeur de la collection New Approaches to Conflict Analysis aux Presses de l'Université de Manchester et co-directeur de la collection Routledge Studies in International Political Sociology.

Julien Pomarède est docteur en sciences politiques et sociales de l'Université libre de Bruxelles. Il y est actuellement chercheur postdoctoral an centre Recherche et études en politique internationale (REPI) et enseigne dans plusieurs universités.

$\mathrm{I}^{1}$ est peu de dire que le secret fascine. La divinité grecque Harpocrate, le corps recouvert d'un long manteau voilant partiellement son visage, les divinités romaines Angerona, bâillon sur la bouche, et Tacita, rendue muette par l'arrachage de sa langue, président au silence et veillent à ce que rien ne sorte du cercle de ceux qui sont instruits des choses mystérieuses et impénétrables ${ }^{1}$. Suivant une expression devenue familière, le mot se fait muet et la bouche demeure cousue. Ce goût pour une réalité dont la représentation se complait en un jeu de cache-cache est une des marques particulières de l'époque maniériste et de l'art baroque ; l'iconographie devient délibérément allusive ${ }^{2}$, le sfumato comme art du brouillage des lignes et la symbolique

1. Dubourdieu A., "Divinités de la parole, divinités du silence dans la Rome antique », Revue de l'Histoire des Religions, vol. 220, n³, 2003, pp. 259-282.

2. Brock M., «Le secret du disegno dans la peinture et dans l'art des fortifications à Venise au 
règnent en maître. Extraire « le secret » de cette gangue fabuleuse, mystique, ésotérique et énigmatique qui l'entoure et qui exerce un pouvoir d'attraction et de fascination n'est pas chose aisée. Il s'agit d'aller à l'encontre d'une esthétique particulière $\mathrm{du}$ secret inscrite au patrimoine de notre environnement politique $^{3}$.

Ce qui est caché, dissimulé ou occulté avec plus ou moins de virtuosité, ce qui ne peut ou ne doit être vu par le profane et qui, par conséquent, ne peut être dévoilé que suivant certaines conditions statutaires ou rites d'initiation reste un puissant ressort éditorial. Nombreux sont les ouvrages qui entendent révéler l'histoire interdite d'un service de renseignement ${ }^{4}$, raconter la vie quotidienne de l'espionnage ${ }^{5}$, pénétrer les coulisses de la diplomatie ${ }^{6}$ et dévoiler avec une satisfaction certaine des situations énigmatiques ${ }^{7}$. Cette dimension « mystérieuse » du secret est également entretenue par le sens savant, ici particulièrement encombrant. Il se déploie généralement sur le mode fonctionnaliste $\mathrm{du}$ «mal nécessaire » car il demeure étroitement associé à la raison d'État ou à l'intime conviction que le sens des responsabilités des groupes dépositaires du secret, et incarnant ici l'exercice du monopole de la violence physique légitime, est avant tout de "savoir se taire » ou de mentir ${ }^{8}$. Par ailleurs, les complexes d'extériorité au milieu investi ainsi que la surdétermination de son homogénéité alimentent en retour ces prénotions et entretiennent l'autodisqualification de l'enquêteur face aux difficultés d'assimilation des codes sociaux et d'acculturation. Dans ces mondes sociaux où le secret est à la fois une règle de conduite intériorisée et un dispositif administratif ordonné et réglementé comme un ensemble de pratiques juridiques, de normes et de technologies concrètes, les modalités d'investigation et la marge de manœuvre scientifique sont, en effet, souvent présentées comme de facto limitées. À la suite des conclusions du forum publié dans la revue ${ }^{9}$, ce numéro entend donc contrer le «défaitisme méthodologique 10 " si récurrent à propos des objets

XVIe siècle », in Dujardin P. (dir.), Le Secret, Lyon/Paris, Presses Universitaires de Lyon, Éditions du CNRS, 1987, pp. 17-30.

3. Gevrey F., Levrier A., Teyssandier B., Éthique, poétique et esthétique du secret de l'Ancien Régime à l'époque contemporaine, Louvain, Peeters Publishers, 2015.

4. Delesse C., NSA. National Security Agency. L'bistoire la plus secrète des agences de renseignement, Paris, Tallandier, 2016 ; Rudgers D., Creating the Secret State: The Origins of the Central Intelligence Agency, 1943-1947, Lawrence, University Press of Kansas, 2000 ; Notin J.-C., Les guerriers de l'ombre, Paris, Tallandier, 2017.

5. Aeberhardt C., Les espionnes racontent, Paris, Robert Laffont, 2017 ; Pelletier E., Dubois C. (dir.), Où sont passés nos espions? Petits et grands secrets du renseignement français, Paris, Albin Michel, 2017.

6. Renaud F., Les diplomates, derrière la façade des ambassades de France, Paris, Nouveau monde éditions, 2011 ; Jauvert V., La face cachée du quai d’Orsay. Enquête sur un ministère à la dérive, Paris, Laffont, 2016.

7. Ganser D., Les armées secrètes de l'OTAN : Réseaux Stay-Behind, Opération Gladio et terrorisme en Europe de l'Ouest, Paris, Éditions Demi-Lune, 2007.

8. Dewerpe A., Espion : une anthropologie historique du secret contemporain, Paris, Gallimard, 1994.

9. Guittet E-P., Pomarède J. (dir.), « Forum : Arpenter les territoires du secret », Cultures $\varepsilon$ Conflits, n¹14-115, 2019, pp. 265-268. 
canoniques des relations internationales : la conduite de la guerre, le champ de la sécurité, les arrangements diplomatiques, les interventions internationales, l'univers du renseignement.

Proposer des clefs d'appréhension du secret dans les enquêtes en sciences sociales n'est, en soi, pas une démarche neuve. De nombreux travaux offrent de riches réflexions méthodologiques sur la place de la discrétion et du secret dans divers secteurs du monde social, comme la religion ${ }^{11}$, la médecine ${ }^{12}$, les banques ${ }^{13}$, la famille ${ }^{14}$, l'inimitié et la sexualité ${ }^{15}$, la consommation de substances illicites ou les mobilités clandestines ${ }^{16}$. Les recherches menées sur ces sujets montrent que le secret n'a rien d'une réponse fonctionnelle à des besoins objectifs et prédéterminés visant à « cacher », " dissimuler » ou "rendre inavouables » certaines pratiques ou situations. Elles insistent également sur la polysémie et la polyphonie du secret : les occultations, non-dits et autres stratégies d'évitement n'ont pas les mêmes ressorts ou effets que les interdits et les tabous. Ces recherches établissent de manière pérenne que le secret est une construction sociale plurielle, aux logiques de fabrication parfois très banales et ordinaires, qui façonne et conditionne une multiplicité d'espaces sociaux et leurs jeux itératifs.

La production des confidentialités dans l'industrie automobile n'est ainsi pas motivée par les mêmes déterminants et rationalités éthiques et disciplinaires que le secret médical, tout comme les confidentialités de ces instances

10. Pouponneau F., La politique française de non-prolifération nucléaire. De la division du travail diplomatique, Bruxelles, Peter Lang, 2015.

11. Bernat C., Puccio-Den D. (dir.), Religion, secret et autorité : Pratiques textuelles et culturelles en clandestinité, Paris, Armand Colin, 2011.

12. Fainzang S., "Secret et mensonge dans le discours médical », Cités, n²6, 2006, pp. 27-36 ; Bessin M., Lechien M.-H., « Hommes détenus et femmes soignantes : l'intimité des soins en prison », Ethnologie française, vol. 32, n¹, 2002, pp. 69-80; Darmon M., Devenir anorexique, une approche sociologique, Paris, La Découverte, 2003.

13. Guex S., "Conflits et marchandages autour du secret bancaire en Suisse à l'issue de la Grande Guerre », L'Année sociologique, vol. 63, n¹, 2013, pp. 157-187; Amicelle A., Berard J., «Vers la fin du secret bancaire ou de la vie privée ? ", Cultures E Conflits, n¹14-115, 2019, pp. 286292.

14. Grobost A., Pour une socio-anthropologie des secrets de famille : l'impact du mythe familial sur la socialisation de l'individu par la révélation d'un secret, Thèse de Doctorat en Sociologie, Université de Franche-Comté, 2012.

15. Goffman E., La Présentation de soi : la mise en scène de la vie quotidienne, Paris, Éditions de Minuit, 1973 ; Bozon M., «Observer l'inobservable : la description et l'analyse de l'activité sexuelle ", in Bajos N., Bozon M., Giami A. (dir.), Sexualité et sida. Recherches en sciences sociales, Paris, ANRS, 1995, pp. 48-49 ; Jovelin E., « De la prostitution aux clients de la prostitution », Pensée plurielle, n²7, pp. 75-92; Darley M., « La prostitution en clubs dans les régions frontalières de la République tchèque ", Revue française de sociologie, vol. $48, \mathrm{n}^{\circ} 2$, 2007, pp. 273-306.

16. Sandberg S., Copes H., "Speaking with ethnographers: The challenges of researching drug dealers and offenders”, Journal of Drug Issues, vol. 43, n¹, 2012, pp. 176-197 ; Khosravi S., “The 'illegal' traveller: an autoethnography of borders”, Social Anthropology, vol. 15, n`3, 2007, pp. 321-334 ; Equoy-Hutin S., Mariani-Rousset S., "Sur les traces du secret : enquête sur la relation de soin et d'accompagnement de l'addiction ", Revue ¿Interrogations?, $\mathrm{n}^{\circ} 22$, 2016, pp. 1-15. 
s'avèrent bien différentes, du fait de leur dimension officielle, validée et légalement codifiée, des secrets produits à partir de pratiques déviantes et au degré d'organisation moins établi, comme l'achat et la prise de drogue ou la prostitution. Les sciences sociales, dans la richesse de leur production, ont ainsi déjà fait émerger la ligne de travail selon laquelle la pluralité ontologique du secret suppose une pluralité d'ordre méthodologique, qui tient compte à la fois des points communs de l'ensemble de ces configurations du secret et de leurs spécificités sociologiques.

Du côté des études de sécurité, le bilan méthodologique sur la question du secret, entendu comme la retenue, le tri et la transmission limitée d'informations relatives aux divers secteurs de la sécurité (police, renseignement, politique étrangère, défense, etc.), n'est, étonnamment, pas si étoffé. Durant la fin des années 1980 et dans les années 1990, les «études critiques de sécurité » (Critical Security Studies) ont participé à un important renouvellement des grilles d'analyse de la sécurité, jusque-là monopolisées, dans le cadre de la Guerre froide, par les grands paradigmes rationalistes des Relations internationales ${ }^{17}$. L'usage des outils de la sociologie politique, alors destinés à comprendre la manière dont les groupes professionnels disent, élaborent, pratiquent et légitiment leurs savoir-faire en matière de sécurité ${ }^{18}$, a permis de dépasser les biais militaro et stato-centrés de l'analyse traditionnelle de la conflictualité. Pourtant, quand bien même les institutions publiques qui se prétendent garantes de l'ordre public fonctionnent sur le principe du secret, ce dernier est relégué au rang des précisions méthodologiques, où les chercheurs expliquent, souvent brièvement et ce même dans un cadre ethnographique, les difficultés rencontrées et les solutions trouvées pour générer une enquête de terrain viable ${ }^{19}$. Des manuels de méthode proposent certes des

17. Buzan B., Waever O., de Wilde J., Security: A New Framework for Analysis, Boulder, Lynne Rienner, 1998 ; Bigo D., "Grands débats dans un petit monde », Cultures E Conflits, n¹920, 1995, pp. 7-48 ; Ashley R., "Living on Border Lines: Man, Poststruturalism, and war", in Der Derian J., Shapiro M., International/Intertextual Relations: Postmodern Readings of World Politics, New York, Lexington Books, 1989, pp. 259-320 ; George J., Discourses of Global Politics: A Critical (Re)Introduction to International Relations, Boulder, Lynne Rienner, 1994.

18. Guittet E.-P. (dir.), «Questions de méthodes. Savoir-faire des études critiques de sécurité », Cultures E Conflits, n`106, 2016 ; Bigo D., « La mondialisation de l'(in)sécurité ? Réflexions sur le champ des professionnels de la gestion des inquiétudes et analytique de la transnationalisation des processus d'(in)sécurisation », Cultures E Conflits, n58, 2005, pp. 53-101; Daho G., «L'érosion des tabous algériens : Une autre explication de la transformation des organisations militaires en France ", Revue française de science politique, vol. 64, $\mathrm{n}^{\circ} 1$, 2014, pp. 5778.

19. Ambrosetti D., « "Décide de demeurer saisi de la question” : La mobilisation du Conseil de sécurité de l'ONU face aux crises ", Cultures E Conflits, $\mathrm{n}^{\circ} 75$, 2009, pp. 99-122; Neumann I., At home with the diplomats: Inside a European foreign ministry Expertise, New York, Cornell University Press, 2012 ; Pouponneau F., «Une division internationale du travail diplomatique : Analyse de la politique étrangère française autour du problème du nucléaire iranien ", Revue Française de Science Politique, vol. 63, n¹, 2013, pp. 51-73 ; Cohen S. (dir.), L'art d'interviewer les dirigeants, Paris, PUF, 1999 ; Buchet de Neuilly Y., L'Europe de la politique étrangère, Paris, Economica, 2005 ; Gusterson H., Nuclear Rites: A Weapons Laboratory at the End of the Cold War, Berkeley, University of California Press, 1998. 
«ficelles » et stratégies d'approche, mais, à nouveau, l'étude du fonctionnement du secret, pourtant essentielle à son appréhension méthodologique, ne constitue pas le centre d'intérêt de ces publications ${ }^{20}$.

Ce paradoxe est d'autant plus marqué au regard de la place importante que le développement contemporain de la lutte contre le terrorisme a donné au secret des politiques de sécurité dans le débat public. À partir de la deuxième moitié des années 2000, Wikileaks dévoile des contenus hautement classifiés sur les méthodes, parfois illégales, de l'armée étatsunienne (tortures et détentions extra-judiciaires, opérations ciblées, dommages collatéraux) ou encore sur les écoutes de hauts responsables politiques européens pratiquées par la National Security Agency (NSA). Cette dernière fera également l'objet de fuites de la part d'Edward Snowden, qui révéla des détails sur des programmes de surveillance de masse aux États-Unis. L' ' Afffaire Snowden » montre ainsi à quel point les nouvelles pratiques technologisées du secret par les professionnels de la surveillance reconfigurent le rapport des autorités publiques à la vie privée 21 . S'ajoute à ces irruptions de la question du secret sur la scène publique leur surexposition médiatique qui participe à sa mise en spectacle et en intrigue.

Que l'on parle de la protection des données sensibles, personnelles et privées dans un monde du tout numérique, de l'usage "d'informations sensibles » par les Cours de justice dans le cadre de procédures concernant des entreprises terroristes ou encore de l'ensemble de ces discussions sur la levée $\mathrm{du}$ secret professionnel dans le cadre de la prévention de la radicalisation, le tandem sécurité/secret est devenu un enjeu de société crucial et se situe au cœur de la reconfiguration de nos espaces politiques. Par conséquent, si nombre de recherches du courant des études critiques de sécurité ont pointé du doigt des évolutions clefs dans l'usage du renseignement, de la surveillance et de l'information dans les cadres d'action contre-terroristes ${ }^{22}$, le secret entourant les pratiques professionnelles n'a guère fait l'objet de réflexions théo-

20. Klotz A., Lynch C. (eds.), Strategies for Research in Constructivist International Relations, New York, M. E. Sharpe, 2007 ; Salter M., Mutlu C. (eds.), Research Methods in Critical Security Studies: An Introduction, Londres, Routledge, 2013 ; Siméant J., Pouponneau F., Lickert V., "Échelles, récifs, bureaux - terrains du politique à l'international », in Siméant J. (dir.), Guide de l'enquête globale en sciences sociales, Paris, CNRS Éditions, 2016, pp. 13-32; Devin G. (dir.), Méthodes de recherche en relations internationales, Paris, Presses de Sciences Po, 2016.

21. Bauman Z., Bigo D., Esteves P., Guild E., Jabri V., Lyon D., Walker R. B. J., « Repenser l’impact de la surveillance après l'affaire Snowden : sécurité nationale, droits de l'homme, démocratie, subjectivité et obéissance ", Cultures E Conflits, nº8, 2015, pp. 133-166.

22. Bonelli L., "Un ennemi "anonyme et sans visage" : Renseignement, exception et suspicion après le 11 septembre 2001 », Cultures E Conflits, n`58, 2005, pp. 101-129; Mattelart A., La globalisation de la surveillance: Aux origines de l'ordre sécuritaire, Paris, La Découverte, 2008 ; Lyon D., "Globalizing Surveillance: Comparative and Sociological Perspectives", International Political Sociology, vol. 19, n², 2004, pp. 135-149 ; Amoore L., "Data Derivatives On the Emergence of a Security Risk Calculus for Our Times", Theory, Culture E Society, vol. 26, n%, 2011, pp. 24-43. 
riques approfondies. Elles n'ont pas non plus procédé à l'objectivation des techniques d'enquête à déployer au sein de ces milieux particulièrement difficiles d'accès.

À partir de recherches empiriques originales et approfondies menées sur des terrains variés, ce numéro de la revue Cultures $\mathcal{E}$ Conflits propose de contribuer à combler ces manques. L'hypothèse de travail centrale de ce numéro repose ainsi sur l'idée d'une "banalisation» du secret de sécurité. Nous proposons de le lire comme une construction sociale dont les mécaniques ont moins à voir avec des logiques «spectaculaires » (comme la protection de données objectivement "très sensibles », "vitales » ou le fait de « cacher » des éléments « compromettants») que d'interactions humaines, d'intérêts et de routines quotidiennes aux variations sommes toutes banales et hétérogènes, mais décisives et structurantes pour comprendre les relations entre champs et groupes sociaux. Poser la question de l'ordinaire du secret est un point d'entrée original pour interroger le quotidien des espaces professionnels et, surtout, comprendre comment il structure l'enquête dans ces milieux. De fait, l'originalité principale de ce numéro, et le vide qu'il entend combler, s'opère par la saisie du secret de sécurité dans le « vif » de son quotidien et de son rôle dans la recherche.

Les mondes de la sécurité ne sont pas habités par un secret homogène, distribué uniformément parmi les populations peuplant ces espaces et délimitant de manière fixe les frontières d'accès aux acteurs et à leurs pratiques. Ce numéro prend le pari inverse en proposant de considérer les effets de la confidentialité comme étroitement dépendants des stratégies d'enquête et d'approche des acteurs et des institutions étudiées. Non pas que le secret n'existe pas ou qu'il existe trop, mais qu'il existe dans une relation sociale ${ }^{23}$. Dès lors, les articles de ce numéro reprennent, chacun à sa manière, trois axes d'analyse principaux.

Le premier concerne la relationnalité méthodologique du secret. En paraphrasant Gilles Deleuze lecteur de Michel Foucault sur le pouvoir, on peut dire que le secret n'a «pas d'essence, il est opératoire, il n'est pas attribut mais relation $24 »$. Autrement dit, la relation de confidentialité est une relation de pouvoir. Le secret ne s'exercerait pas de manière prédéterminée sur l'enquêteur, mais s'activerait dans la relation que ce dernier construit avec l'objet qu'il étudie. Conçu comme relation, le secret de sécurité s'entrevoit alors comme un phénomène multiple plutôt que comme un horizon homogène indépassable. En effet, le secret peut, comme il en sera question dans ce dossier, servir à durcir une relation de pouvoir fondée sur la différence de niveau ou de posi-

23. De Goede M., Bosma E., Pallister-Wilkins P., "Introduction: Navigating secrecy in security research", in De Goede M., Bosma E., Pallister-Wilkins P. (eds.), Secrecy and Methods in Security Research: A Guide to Qualitative Fieldwork, Londres, Routledge, 2019, p. 7.

24. Deleuze G., Foncault, Paris, Les Éditions de Minuit, 1986, p. 34. 
tion sociale entre l'enquêteur et l'enquêté. Il y a là beaucoup à apprendre de la littérature méthodologique, qui a mis au jour des formes d'asymétrie des relations très diverses, de niveau social ${ }^{25}$, de genre ${ }^{26}$, ethniques ${ }^{27}$, entre le chercheur et les acteurs. Il convient ainsi de croiser ce corpus à la question du secret afin d'épuiser certaines des configurations possibles d'enquête.

Concrètement, cette relation prend d'abord forme dans une distinction dans l'interaction enquêteur/enquêté. La forme d'asymétrie la plus évidente, mise en avant dans ce numéro, que produit le secret entre l'acteur et le chercheur est probablement la « détention » supposée, par le premier, d'informations que le second cherche à obtenir. De fait, la confidentialité a pour première fonction de constituer une barrière derrière laquelle les enquêtés se placent pour justifier leur silence. Or, cette relation, au départ d'opposition, peut évoluer, au gré d'une confiance minimale entre l'initié et le profane, au fur et à mesure de l'accès à l'information. Elle peut reposer sur des stratagèmes plus ou moins conscientisés (ruse, diversion, dissimulation/omission, bluff, flatterie, chantage et name droping ${ }^{28}$ ). Ajoutons que les rôles sont eux-mêmes fluctuants : il arrive fréquemment que l'enquêteur se retrouve en position d'enquêté ${ }^{29}$, si bien que la tension affecte tout aussi bien l'initié : il ne doit pas divulguer, compromettre, trahir ${ }^{30}$. Le dossier montre que la relation de l'enquêteur au secret peut aussi prendre des formes diverses : la (re)formulation de la question de recherche (qui permet de surmonter l'obstacle de données impossibles à récolter), les types d'acteurs visés et les modes de production des confidentialités plus ou moins solides qui les entourent, la garantie d'un anonymat dans le report de leurs propos, l'asymétrie enquêteur/enquêté en

25. Daho G., «Faire parler les généraux. Retour sur quelques usages des techniques d'entretien en milieux militaires ", Revue ¿̇nterrogations?, $\mathrm{n}^{\circ} 22$, 2016, pp. 1-12 ; Bruneteaux P., « Les politiques de l'urgence à l'épreuve d'une ethnobiographie d'un SDF ", Revue Française de Science Politique, vol. 57, n¹, 2007, pp. 47-67 ; Chamboredon H., Pavis F., Surdez M., Willemez L., "S'imposer aux imposants : À propos de quelques obstacles rencontrés par des sociologues débutants dans la pratique et l'usage de l'entretien », Genèses, vol. 16, n¹, 1994, pp. 114-132 ; Pinçon M., Pinçon-Charlot M., «Pratiques d'enquête dans l'aristocratie et la grande bourgeoisie : distance sociale et conditions spécifiques de l'entretien semi-directif », Genèses, vol. 3, n ${ }^{\circ}, 1991$, pp. 120-133.

26. Cohn C., "Sex and Death in Rational World of Defense Intellectuals", Signs, vol. 12, n ${ }^{\circ} 4$, 1987, pp. 687-718 ; Vannetzel M., "À la frontière du parti : jeux d'inclusion et d'exclusion d'une chercheuse chez les Frères musulmans égyptiens ", Revue internationale de politique comparée, $\mathrm{n}^{\circ} 17,2010$, pp. 47-62.

27. Boumaza $M$, «L'expérience d'une jeune chercheuse en "milieu extrême" : une enquête au Front National », Regards sociologiques, n²2, 2001, pp. 105-121 ; Poiret C., " Pour une approche processuelle des discriminations : entendre la parole minoritaire », Regards sociologiques, n`39, 2010, pp. 5-20; Simon P., « Les statistiques, les sciences sociales françaises et les rapports sociaux ethniques et de "race" ", Revue française de sociologie, n49, 2008, pp. 153162.

28. Laurens S., Frédéric N., Enquêter: de quel droit? Menaces sur l'enquête en sciences sociales, Bellecombe en Bauges, Le Croquant, 2010.

29. Dauvin P., Siméant J., "Travailler sur l'humanitaire par entretiens - Retour sur une "méthode" ", Mots, vol. 65, n¹, 2001, pp. 117-133.

30. Simmel G., "Le secret et la société secrète ", in Sociologie : Études sur les formes de la socialisation, Paris, PUF, 1999, pp. 347-405. 
termes de positions sociales, de trajectoires et de connaissance des pratiques émiques ou encore dans les temporalités (dis)continues d'accès aux espaces étudiés.

Par conséquent, les contributeurs au numéro s'attacheront certes à déconstruire les effets de contrainte liés à la relationnalité méthodologique du secret, mais aussi les opportunités et ressources que présente ce dernier. Alors qu'une lecture substantialiste du secret aurait tendance à concevoir celui-ci sous l'angle de ce qu'il empêche et limite dans l'enquête, la vision relationnelle adoptée perçoit le secret comme une dynamique génératrice d'où peuvent aussi découler des avantages dans des logiques de situation que l'on présenterait spontanément comme défavorables à l'enquêteur. Dès lors que le secret est conçu comme un rapport social contingent et construit, il peut être vu, dans une certaine mesure, comme un artefact qui permet des ouvertures et donc l'accès aux données. Pour l'exprimer brièvement, la confidentialité constituerait aussi un levier à actionner, que cela soit, par exemple, dans la liberté de prise de parole que peut paradoxalement s'octroyer, sur le mode de l'hypo-correction, un acteur se percevant comme maîtrisant parfaitement les codes du dicible et du non-dicible, dans l'obtention des habilitations de sécurité au vu d'une immersion ethnographique, qui garantit un accès légal, reconnu et permanent au lieu étudié, ou encore dans le fait de jouer sur les conflictualités et solidarités entre différents acteurs pour les « pousser » à la confidence avec pour risque pour le chercheur un dévoilement de ses hypothèses ou une compromission auprès des enquêtés sous forme "d'échange de bons procédés ».

Deuxièmement, toujours dans une perspective relationnelle, le numéro donne des éléments de réponse à la question du secret dans le déroulement des entretiens. Lorsque les travaux se penchent sur les rapports de domination au cours des entretiens, ils tendent à objectiver la «culture du secret » comme trait distinctif des groupes dominants. D’un côté, l'approche réflexive repose sur l'intériorisation de la violence symbolique immanquablement produite par un rapport de domination subi par l'enquêteur ${ }^{31}$ : si elle présente le mérite de refuser le particularisme de l'interaction enquêteur/enquêté par rapport aux autres formes de relations sociales a priori déséquilibrées, elle confine à l'autodisqualification, car du fait de l'inertie de l'babitus, l'enquêteur commettra toujours des « gaffes » trahissant son origine sociale, et donc son extériorité au milieu. À nouveau, il y a ici beaucoup à apprendre d'autres littératures en sciences sociales qui se sont intéressées au secret, et plus spécifiquement à ses usages dans les groupes les plus dominés ou déviants de l'espace social. Il en ressort que le secret est loin d'être l'apanage des dominants et que les difficultés méthodologiques relatives au secret ne sont guère plus difficiles à surmonter dans des entretiens avec des fonctionnaires, diplomates, militaires hauts- 
gradés, industriels de l'armement, ou agents de sécurité, bien au contraire. De l'autre côté, l'approche stratégique réfutant la fatalité de la distance sociale, s'appuie sur l'anticipation des interactions érigée en « art 32 » : si elle souligne la dimension relationnelle du pouvoir, elle tend à l'auto-satisfaction de l'enquêteur, qui soit tient là une " revanche sociale » soit se méfie trop peu des risques de connivence. Les articles du dossier montrent, chacun à sa façon, les limites de la dichotomie méthodologique « réflexive/stratégique », en mettant au jour à la fois les pesanteurs structurelles et objectives qui pèsent sur l'enquêteur en entretien (statut d'outsider, genre, mises en scène du secret de sécurité), ainsi que les divers procédés par lesquels ces contraintes peuvent être, si ce n'est dépassées, au moins négociées et appréhendées pour produire une recherche viable et précise.

Troisièmement, ce numéro montre que le secret joue dans la délimitation des espaces professionnels et met en avant des procédés méthodologiques pour tirer parti de cette dimension constitutive du secret dans les milieux étudiés. Le secret constitue alors une ressource qui renseigne sur le fonctionnement de l'environnement analysé. Le secret ne fait pas qu'exclure. Dans leur ouvrage retraçant les variations historiques du « partage du secret », Bernard Darbord et Agnès Delage montrent avec précision les différences d'emploi entre le champ lexical pour dire ce que l'on cache (le secret par exclusion, les exercices de la tromperie et les formes de dissimulation), et celui pour dire ce que l'on montre avec discrétion (le secret partagé, l'intimité, la confiance et ses formes de contrôle) ${ }^{33}$. Si le secret est excluant, il est tout aussi créateur de lien. Parmi les acteurs qui pratiquent le secret, celui-ci constitue un phénomène intégrateur et soude. Le sociologue Simmel a été le premier à discuter le secret en ce sens ${ }^{34}$. C'est là que s'ouvre un autre angle mort de la réflexivité méthodologique sur le secret de la sécurité : que se passe-t-il lorsque le chercheur entre enfin dans la confidence ? Est-il invité aux réunions, mis dans la boucle des échanges de mails ? À l'inverse, se sent-il redevable ou même coupable de connivence ? Le secret brouille et consolide à la fois les frontières des espaces professionnels étudiés. Le numéro met en avant le fait que l'exercice du secret est une tâche extrêmement parcellisée qui donne à voir nombre de cloisonnements politiques, technologiques et organisationnels. Les pratiques quotidiennes, individuelles et collectives, informelles et institutionnelles liées à la divulgation ou à la protection du secret renseignent sur les formes de structuration comme la solidarité organique, la différenciation et les luttes entre services.

32. Cohen S. (dir.), op.cit., 1999 ; Crane-Seeber J.P., "Learning by feeling”, in Salter M., Mutlu C. (eds.), Research Methods in Critical Security Studies: An Introduction, Londres, Routledge, 2013, pp. 72-75 ; Vrasti W., "The Strange Case of Ethnography and International Relations”, Millenium - Journal of International Studies, vol. 37, n², 2008, pp. 279-301.

33. Darbord B., Delage A. (dir.), Le partage du secret. Cultures du dévoilement et de l'occultation en Europe, du Moyen Âge à l'époque moderne, Paris, Armand Colin, 2013.

34. Simmel G., op. cit. 
Le numéro s'ouvre avec l'article de Florent Pouponneau. Démontrant de manière précise qu'il n'y a aucune raison de céder au défaitisme méthodologique face aux terrains de la sécurité internationale, l'auteur déconstruit ce qu'il identifie comme la «mise en scène du secret d'État » et expose les procédés méthodologiques qu'il a mis en place pour analyser, en particulier, l'évolution des luttes bureaucratiques ayant structuré la position de la France sur la question de la non-prolifération nucléaire. F. Pouponneau revient notamment sur trois réflexions : l'usage des sources ouvertes « non-habituelles » (mémoires, enquêtes journalistiques, blogs, mémos administratifs, câbles Wikileaks), la négociation des situations d'entretien et la valeur analytique finalement relative des données très classifiées.

Le second article du dossier est de Julien Pomarède. Dans ce texte, l'auteur évoque son observation participante de plusieurs mois au Quartier général de l'Organisation du Traité de l'Atlantique Nord (OTAN), dans le cadre d'une recherche sur les politiques contre-terroristes post-11 septembre 2001 de l'OTAN. Ce faisant, J. Pomarède traite d'une modalité spécifique de production du secret en enquête, à savoir l'expérience ethnographique. Partant du constat que les Études critiques de sécurité ont tendance à réduire l'ethnographie à un « héroïsme méthodologique » qui met en avant les qualités individuelles du chercheur, l'auteur se penche sur les facteurs davantage structurels qui pèsent sur une enquête conduite de l'intérieur. Le texte met en avant deux dimensions : la négociation du statut dual d'insider (stagiaire)/outsider (chercheur) dans le quotidien de l'observation et la manière dont l'environnement bureaucratique (en termes de jeux d'interconnaissances et de confidentialité des données recueillies) s'appréhende.

Le troisième article du numéro est de Catherine Hoeffler. À partir d'une recherche sur un terrain d'enquête très masculinisé, les politiques d'armements, C. Hoeffler propose une réflexion originale sur un véritable angle mort : le rapport du genre au secret dans la conduite de l'enquête. En décloisonnant et en discutant la littérature féministe sur les modes de production genrées du savoir scientifique, elle avance deux arguments. Le premier est que la relation au secret dans l'enquête est partiellement structurée par des relations de genre, qui se manifestent largement, sur les terrains de la sécurité, par une ascendance masculinisante qui influence l'enquête. Le second est que la relation secret-genre n'est pas réductible à la dichotomie masculin-féminin mais prend forme, et se négocie, à travers d'autres variations ou rôles endossés par l'enquêtrice (jouer la compétence ou l'ignorance, la carte du doctorat ou du journalistique).

Le texte de Karine Côté-Boucher et Ariane Galy constitue le quatrième article du dossier. Plus qu'une réflexion méthodologique, cet article propose une véritable immersion dans les usages professionnels du secret. À partir de 
leurs recherches sur les pratiques et l'industrie du contrôle frontalier en Amérique du Nord, les auteures montrent la place centrale du secret dans les reconfigurations de ces mondes professionnels, en particulier dans le cadre de leur technologisation croissante. En explicitant les limites des approches intentionnalistes du secret, qui se résument à l'analyse des intérêts à détenir telle ou telle information, les auteures mettent au jour la fonctionnalité sociale et professionnelle de situations où le secret se dilue dans un vaste ensemble de technologies et d'automatisation du contrôle aux frontières (où la distribution de l'information revêt des contours forts imprécis). Cette complexification techno-centrée du contrôle frontalier et de la production du secret introduit des changements profonds à la fois dans la hiérarchie des groupes professionnels et dans le degré de déresponsabilisation des autorités publiques dans le durcissement des politiques d'entrée.

Ce numéro de la revue est aussi l'occasion d'accueillir un article en horsthème. À partir d'une série d'entretiens réalisés avec des hauts fonctionnaires turcs et des diplomates européens, Shoshana Fine offre une analyse des raisons de l'alignement de ces hauts fonctionnaires turcs sur la politique migratoire européenne, mais aussi toute une réflexion sur les dimensions routinières, ordinaires et symboliques de la fabrication de politiques internationales. Cet article est certes un hors-thème, mais il s'inscrit pleinement dans la continuité des travaux publiés sur le sujet dans la revue depuis des années et contribue à nourrir la réflexion autour d'une politique ô combien d'actualité.

Ce numéro comporte aussi deux recensions d'ouvrages portant sur les conflits, les instruments de la paix et le devenir des anciens combattants dans deux contextes différents. Adélaïde Martin propose une analyse de l'ouvrage de Daniel Bultmann, The Social Order of Postconflict Transformation in Cambodia: Insurgent Patbways to Peace (Lanham, Lexington Books, 2018) et Valérie Rosoux consacre sa recension à l'ouvrage de Nathalie Duclos, Courtiers de la paix: Les vétérans au coeur du state building international au Kosovo (Paris, CNRS Éditions, 2018).

Finalement, le dossier de la rubrique Regards sur l'entre-deux est consacré au premier film de Chowra Makaremi «Hitch, une histoire iranienne ». Ce dossier nous permet de poursuivre une réflexion menée de longue date sur les logiques et les effets de certaines formes de violence dont les disparitions de personnes et les imbrications de l'intime et du politique. 